\title{
Mesothelial cells facilitate cancer stem-like properties in spheroids of ovarian cancer cells
}

\author{
AKEMI SHISHIDO $^{1}$, SEIJI MORI ${ }^{2}$, YUHKI YOKOYAMA ${ }^{1}$, YOSHINOSUKE HAMADA ${ }^{1,3}$, KAZUMASA MINAMI $^{4}$ \\ YAMIN QIAN $^{1}$, JIAQI WANG ${ }^{1}$, HARUKA HIROSE ${ }^{1}$, XIN WU $^{1}$, NAOMASA KAWAGUCHI ${ }^{1}$, \\ SACHIKO NAGUMO $^{1}$, NARIAKI MATSUURA ${ }^{1,5}$ and HIROFUMI YAMAMOTO ${ }^{1}$ \\ ${ }^{1}$ Department of Molecular Pathology, Division of Health Sciences, Graduate School of Medicine, Osaka University, \\ Suita, Osaka 565-0871; ${ }^{2}$ Department of Medical Technology, Faculty of Health Sciences, \\ Morinomiya University of Medical Sciences, Osaka City, Osaka 559-8611; ${ }^{3}$ Department of Pediatric Dentistry, \\ Osaka Dental University, Hirakata, Osaka 573-1121; ${ }^{4}$ Department of Medical Physics and Engineering, \\ Division of Health Sciences, Graduate School of Medicine, Osaka University, Suita, Osaka 565-0871; \\ ${ }^{5}$ Osaka International Cancer Center, Osaka City, Osaka 541-8567, Japan
}

Received December 10, 2017; Accepted May 9, 2018

DOI: 10.3892/or.2018.6605

\begin{abstract}
Ovarian cancer is characterized by widespread peritoneal dissemination with ascites. Spheroids observed in the ascites of ovarian cancer patients are a mixture of cancer cells and mesothelial cells. In the present study, we evaluated whether mesothelial cells exfoliated from the peritoneum facilitate tumor spheroid formation and give rise to cancer stem-like properties in ovarian cancer cells. Spheroids from the CAOV3 and A2780 ovarian cancer cell lines grew much larger in co-culture with mesothelial cells than in monoculture under 3D conditions. The spheroids in co-culture displayed high $\mathrm{Ki}-67$ expression in the peripheral zone and low expression in the central zone area. The expression of CD133 emerged in the inner portion of spheroids at later time-points (96 and $168 \mathrm{~h}$ ), indicating that cancer cells expanded to the inner spheroid and acquired stem cell-properties. The mRNA levels of cancer stem cell markers Dclk-1, CD44 and Bmi-1 significantly increased in co-cultured CAOV3 and mesothelial cells compared to CAOV3 cells alone. Furthermore, the mesothelial cells promoted the tumorigenesis and growth of the CAOV3 cells in a mouse xenograft model compared to
\end{abstract}

Correspondence to: Professor Hirofumi Yamamoto, Department of Molecular Pathology, Division of Health Sciences, Graduate School of Medicine, Osaka University, 1-7 Yamadaoka, Suita, Osaka 565-0871, Japan

E-mail: hyamamoto@sahs.med.osaka-u.ac.jp

Professor Seiji Mori, Department of Medical Technology, Faculty of Health Sciences, Morinomiya University of Medical Sciences, 1-26-16 Nankokita Suminoe, Osaka City, Osaka 559-8611, Japan

E-mail: s-mori@morinomiya-u.ac.jp

Key words: ovarian cancer, spheroid, mesothelial cells, carcinomatous peritonitis, cancer stem cell, CD133 cancer cells alone. In conclusion, mesothelial cells promoted spheroid formation by ovarian cancer cells and facilitated cancer stem-like properties.

\section{Introduction}

Ovarian cancer is the leading cause of death from gynecological cancers. Approximately $50-85 \%$ of patients experience recurrence within 5 years and the median survival time after recurrence is approximately 2 years (1-3). One of the reasons for this poor prognosis is disseminated disease $(4,5)$. Serous carcinoma of surface epithelial-stromal tumors, which is the major histological type, often appears in the ascites and results in peritoneal dissemination to the pelvis and abdomen (6-8). Patients eventually die from cachexia or bowel obstruction due to the large intraperitoneal tumor burden (9). For peritoneal dissemination, carboplatin and paclitaxel are effective as an initial treatment in advanced ovarian cancer patients $(1,3)$. However, most patients later present chemoresistance.

During daily workups for cytopathological diagnosis, we have noticed that flat mesothelial cells are often accompanied by ovarian cancer cell clusters in the ascites. Mesothelial cells are the major constituent of the peritoneum covering the superficial area. This cell type may be involved in the formation of peritoneal metastasis by producing hyaluronic acid and various kinds of extracellular matrix (ECM) and adhesion molecules (10). Thus, ovarian cancer cells are thought to adhere to the peritoneum via $\beta 1$ integrins, hyaluronic acid, and CD44. In addition, the ECM exists in the connective tissues of the subserosa under the mesothelial cell layer (11-15).

To explore the genesis of peritoneal dissemination, many studies have been performed to clarify the mechanism by which cancer cells attach to the peritoneum, however few studies have investigated floating ovarian cancer cell clusters in ascites. Ovarian cancer cells in ascites usually form a papillary spheroid-like cell cluster and ball pattern (disco or mirror ball pattern), and the cells proliferate in suspension. 
Burleson et al reported that ovarian tumor spheroids contain both mesothelial and inflammatory cells (12), suggesting that the interaction between ovarian cancer cells and mesothelial cells in the spheroid may be important for tumor progression. However, the details of this mechanism remain largely unknown.

These cells in spheroids appear to acquire some specific ability to proliferate and survive devoid of tumor neovascularization in ascitic fluid. As spheroid formation is a putative feature of cancer stem cells (CSCs), we hypothesized that mesothelial cells may play a role in the development of tumor spheroids and give rise to cancer stem-like properties in ovarian cancer cells. CSCs have been identified in many types of solid tumors and are relatively quiescent, can self-renew, grow as spheroids and make up the tumor bulk by generating differentiated daughter cells through asymmetric division $(16,17)$. To address this hypothesis, we investigated the role of mesothelial cells exfoliated from the rat peritoneum in the formation of ovarian cancer spheroids using a three-dimensional (3D) culture system and examined whether it is linked to cancer stem-like properties.

\section{Materials and methods}

Cell culture. Human ovarian cancer cell lines CAOV3, A2780, and SKOV3 and human Met-5A mesothelium cell line were obtained from the American Type Culture Collection (ATCC; Manassas, VA, USA). The A2780 and SKOV3 cells were grown in RPMI-1640 (Thermo Fisher Scientific, Inc., Waltham, MA, USA) supplemented with $10 \%$ fetal bovine serum (FBS; Thermo Fisher Scientific, Inc.), $2 \mathrm{mM}$ L-glutamine and $50 \mathrm{U} / \mathrm{ml}$ penicillin $\mathrm{G} /$ streptomycin at $37^{\circ} \mathrm{C}$ in a humidified $5 \% \mathrm{CO}_{2}$ atmosphere. CAOV3 cells were grown in Dulbecco's modified Eagle's medium (DMEM; Thermo Fisher Scientific, Inc.) supplemented with 10\% FBS, $2 \mathrm{mM}$ L-glutamine and $50 \mathrm{U} / \mathrm{ml}$ penicillin $\mathrm{G} /$ streptomycin at $37^{\circ} \mathrm{C}$ in a humidified $10 \% \mathrm{CO}_{2}$ atmosphere. Met-5A cells were cultured in DMEM/F12 medium (Nacalai Tesque, Inc., Kyoto, Japan) supplemented with $10 \% \mathrm{FBS}, 5 \mathrm{ng} / \mathrm{ml}$ EGF, hydrocortisone $(0.4 \mu \mathrm{g} / \mathrm{ml})$, hydrocortisone $(0.1 \mu \mathrm{g} / \mathrm{ml})$, insulin $(2.5 \mu \mathrm{g} / \mathrm{ml}), 50 \mathrm{U} / \mathrm{ml}$ penicillin/streptomycin at $37^{\circ} \mathrm{C}$ in a $5 \% \mathrm{CO}_{2}$ atmosphere. A2780, CAOV3 and SKOV3 cells were transfected with $\mathrm{pEGFP}$ vector to distinguish between ovarian cancer cells and mesothelial cells in culture. Green fluorescent protein (GFP)-positive cells were selected by G418 to establish stable cell lines.

Isolation and propagation of rat mesothelial cells. Rat mesothelial cells were isolated from the omentum and mesenterium of 8-week-old female Sprague-Dawley rats (Charles River Laboratories Japan, Inc., Yokohama, Japan) according to a method previously described $(18,19)$. Briefly, the peritoneum was washed with phosphate-buffered saline (PBS) and incubated with $0.05 \%$ trypsin/EDTA (Nacalai Tesque Inc.) at $37^{\circ} \mathrm{C}$ for $50 \mathrm{~min}$. The cells peeled from the peritoneum were recovered and cultured on a dish coated with $0.1 \%$ type 1 collagen at $37^{\circ} \mathrm{C}$ in a $5 \% \mathrm{CO}_{2}$ atmosphere. The cells were passaged every 3-4 days and used for assays. Mesothelial cell traits were confirmed by immunohistochemical staining for calretinin and $\alpha$-smooth muscle actin ( $\alpha$-SMA).
Spheroid culture. Spheroids were generated on plates coated with $40 \%$ poly 2-hydroxyethyl methacrylate (Poly-HEMA; Sigma-Aldrich, St. Louis, MO, USA). Ovarian cancer cell lines CAOV3, A2780 and SKOV3 (GFP-labeled, 2x10 ${ }^{5}$ ) and rat mesothelial cells or Met-5A human mesothelium cells $\left(2 \times 10^{5}\right)$ were mixed and cultured on Poly-HEMA-coated plates for different durations. Parallel monocultures of ovarian cancer cells or rat mesothelial cells were prepared as a control.

Immunohistochemical staining. The harvested spheroids were fixed in $10 \%$ neutral buffered formalin for 24 to $48 \mathrm{~h}$. Spheroids were suspended in PBS and centrifuged to form a pellet in the bottom of a microtube. The supernatant was removed and $0.1 \%$ agarose (Nacalai Tesque, Inc.) was overlaid in a microtube. Paraffin penetration of solidified agarose containing spheroids was performed in Tissue-Tek VIP (Sakura Finetek Japan Co., Ltd., Tokyo, Japan).

Sections ( 2 to $3-\mu \mathrm{m}$ thick) were stained with the following antibodies at $4^{\circ} \mathrm{C}$ overnight: Anti-calretinin (rabbit polyclonal, 1:1,000; cat. no. PA5-16681; Thermo Fisher Scientific, Inc.), anti- $\alpha$-SMA (1A4, mouse monoclonal, 1:100; cat. no. ab7817; Abcam, Cambridge, UK), anti-Ki-67 (MIB-1, mouse monoclonal, 1:1,000; cat. no. M724029; Agilent Technologies, Santa Clara, CA, USA), anti-ALDH1/2 (H-8, mouse monoclonal, 1:5,000; cat. no. sc-166362; Santa Cruz Biotechnology, Inc., Dallas, TX, USA), anti-CD44 (EPR1013Y, rabbit monoclonal, 1:400; cat. no. ab51037; Abcam) and anti-CD133 (W6B3C1, mouse monoclonal, 1:400; cat. no. 130-092-395; Miltenyi Biotec, Bergisch Gladbach, Germany). After sections were deparaffinized, antigen retrieval was performed using a high pressure chamber (Agilent) at $121^{\circ} \mathrm{C}$ for 2 min to unmask the epitopes in $0.1 \mathrm{~mol} / 1$ citric acid buffer ( $\mathrm{pH} 7.4)$. An Elite ABC kit (Vector Laboratories, Inc., Burlingame, CA, USA) was used for the immunostaining and the antibody complex was visualized by 3,3'-diaminobenzidine tetrahydrochloride (DAB).

$R N A$ extraction and $q R T-P C R$. Total RNA was collected from cultured cells using Sepasol-RNA I Super G (Nacalai Tesque) and complementary DNA synthesized from $1.0 \mu \mathrm{g}$ of total RNA using oligo (dT) primer and a Reverse Transcription System (Promega, Madison, WI, USA) according to the manufacturer's instructions. Quantitative PCR was performed with specifically designed oligonucleotide primers and LightCycler FastStart DNA Master SYBR Green I using a LightCycler 2.0 instrument (Roche Diagnostics, Mannheim, Germany). The expression of the target gene was normalized relative to GAPDH mRNA expression. The primers used were: GAPDH forward, 5'-CAACTACATGGTTTACATGTTC-3' and reverse, 5'-GCCAGTGGACTCCACGAC-3'; CD44s forward, 5'-ATA ATAAAGGAGCAGCACTTCAGGA-3' and reverse, 5'-ATA ATTGTGTCTTGGTCTCTGGTAGC-3'; Dclk-1 forward, 5'-AGTCTTCCGATTCCGAGTTGAG-3' and reverse, 5'-CAG CAACCAGGAATGTATTGGA-3'; Bmi-1 forward, 5'-TGT AAAACGTGTATTGTTCGTTAC-3' and reverse, 5'-CAA TATCTTGGAGAGTTTTATCTGACC-3'.

Xenograft experiments. Ten, 5-week old female SCID-Beige (CB17.Cg-PrkdcscidLystbg-J/CrlCrlj) mice (16.2 g average body weight) were obtained from Charles River Laboratories. 
A

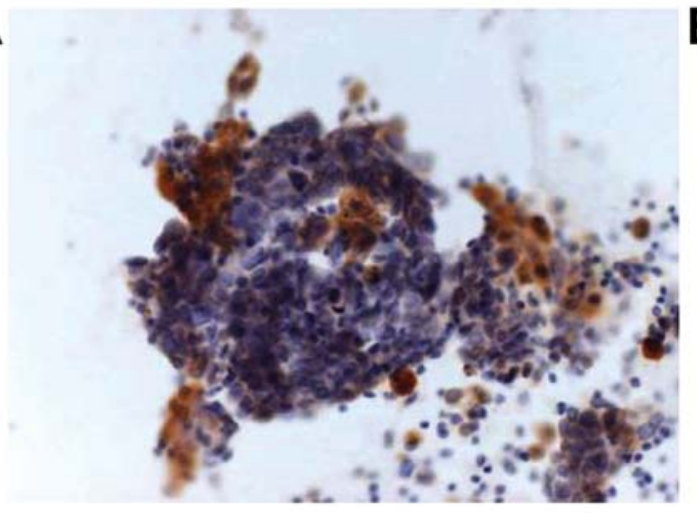

B

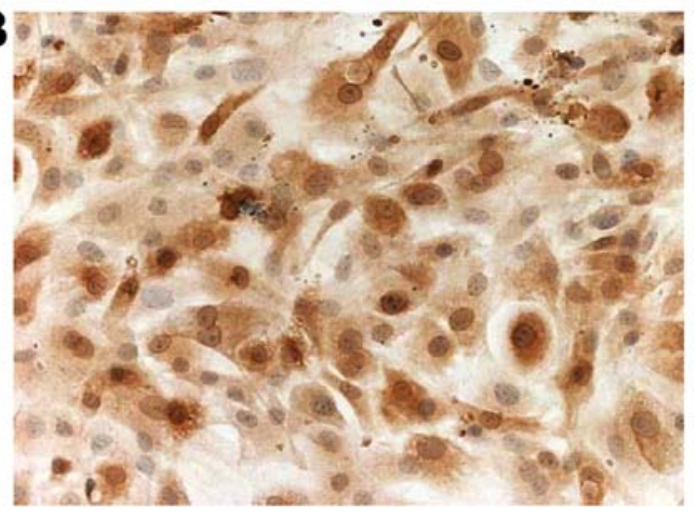

Figure 1. Calretinin immunostaining. (A) Clinical sample of ovarian cancer cell spheroids from a patient with carcinomatous peritonitis. Magnification, x100. This sample was taken for pathological diagnosis by abdominal puncture. (B) Mesothelial cells isolated from the rat abdominal cavity. Magnification, $\mathrm{x} 400$.

Food, and water were autoclaved and changed regularly. All the mice were bred and maintained in SPF condition at $23^{\circ} \mathrm{C}$ in a daily cycle of $12 \mathrm{~h}$ light and $12 \mathrm{~h}$ darkness at Osaka University Graduate School of Medicine, Division of Health Sciences, in a controlled state. An equal number of CAOV3 cells and rat mesothelium cells $\left(1 \times 10^{6}\right.$ cells) were cultured on Poly-HEMA-coated dishes for $24 \mathrm{~h}$. The cells were collected and suspended in 50\% Matrigel (Corning, Corning, NY, USA) in a total volume of $140 \mu \mathrm{l}$ and then subcutaneously injected into the lower backs of mice. Tumor growth was monitored using calipers and tumor volume (v) was calculated as follows: $\mathrm{v}=\left(\mathrm{a} \times \mathrm{b}^{2}\right) / 2$, where $\mathrm{a}$ is the maximum tumor axis and $\mathrm{b}$ the length of the minor axis. All experiments using mice were approved by the Institutional Animal Care and Use Committee Osaka University Graduate School of Medicine and the Committee for the Ethics of Animal Experiments of Osaka University (approval no. 28-03-001).

Statistical analysis. Data are presented as the mean \pm SEM. The statistical significance of differences between two groups was calculated by the Student's t-test. When more than two groups were compared, one-way ANOVA was used followed by Bonferroni's multiple comparison test to determine the statistical significance of the differences. Statistical analyses were performed using the JMP 12 software (SAS Institute, Cary, NC, USA) and GraphPad Prism version 6.00 for Mac (GraphPad Software, San Diego, CA, USA). $\mathrm{P}<0.05$ was considered to indicate a statistically significant difference.

\section{Results}

Calretinin immunostaining. A clinical sample of the spheroids was obtained from ascitic fluid by abdominal puncture in a 77-year old female patient. Pathological diagnosis indicated that the spheroid was composed of ovarian cancer cells and the patient was diagnosed with carcinomatous peritonitis. Immunohistochemistry revealed that calretinin, a marker of mesothelial cells (20), was partially expressed in the spheroids (Fig. 1A). Subsequently, we assessed the purity of mesothelial cells isolated from the rat abdominal cavity by immunostaining for calretinin. The cells were positive in the nucleus and cytoplasm to varying extents (Fig. 1B).
Mesothelial cells promoted in vitro spheroid formation in CAOV3 and A2780 ovarian cancer cells. In the 3D cultures on poly-HEMA-coated plates, GFP-labeled CAOV3, A2780 and SKOV3 cells were grown in the absence or presence of rat mesothelial cells. The spheroids grew much larger in the presence of mesothelial cells plus CAOV3 or A2780 cells compared to cancer cells alone. Conversely, the SKOV3 cells formed large spheroids even in the absence of mesothelial cells (Fig. 2A). The time course studies at 24, 48, 72 and $120 \mathrm{~h}$ are displayed in Fig. 2B-a-c. H\&E staining of the 3D cultures of rat mesothelial cells is also displayed in Fig. 2B-d.

Assessment of the proliferative activity using Ki-67 antibody. To examine the proliferative activity of cells, cell blocks were made at the indicated time-points. Ki-67-positive cells were evaluated in monoculture (cancer cells alone) and co-culture (cancer cells plus mesothelial cells) conditions. In monocultures of CAOV3 and A2780 cells, Ki-67 positivity was randomly observed, whereas the expression of $\mathrm{Ki}-67$ was high in the peripheral zone and low in the central area of spheroids in co-culture (Fig. 3A and B). In SKOV3 cells, proliferative cells were randomly distributed in monoculture spheroids (Fig. 3C). Conversely, the co-culture spheroids had Ki-67-positive cells at the peripheral zone at 48 and $72 \mathrm{~h}$ (Fig. 3C).

Immunostaining of CSC markers. The majority of CAOV3 cells in monoculture exhibited intense expression of CD44 and ALDH1/2 and positive staining was observed at the periphery of spheroids at $24 \mathrm{~h}$ in co-culture with mesothelial cells (Fig. 4A). At subsequent time-points, the expression of CD44 and ALDH1/2 continued and extended to the whole spheroid body (Fig. 4A). The CAOV3 cells exhibited weak CD133 expression in both monoculture and co-culture at 24-72 h (Fig. 4A). However, intense expression of CD133 was observed in the cell membrane in the inner portion of spheroids at $96 \mathrm{~h}$ (Fig. 4B-a) and $168 \mathrm{~h}$ (Fig. 4A-c). Ki-67 staining revealed that the proliferative activity of the cancer cells was high at the periphery and low in the inner portion of spheroids at $96 \mathrm{~h}$ (Fig. 4B-a). Staining mesothelial cells with $\alpha$-SMA antibody revealed that they were located in the central area at $48 \mathrm{~h}$ and then shrunk (144 h) as the cancer cells expanded (Fig. 4B-b). Calretinin staining of mesothelial cells also revealed concordant results at 48 and 168 h (Fig. 4B-c). 

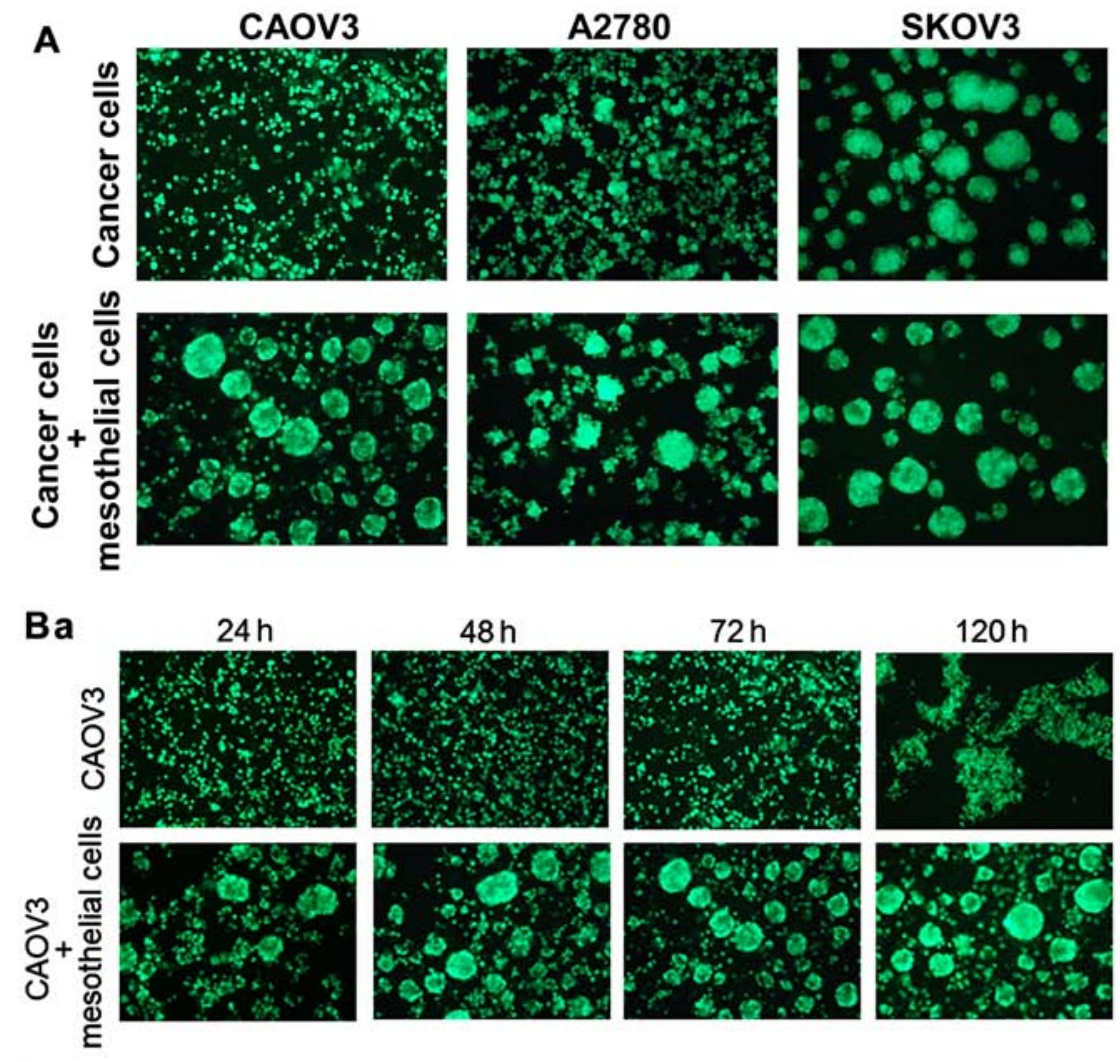

b

$24 \mathrm{~h}$

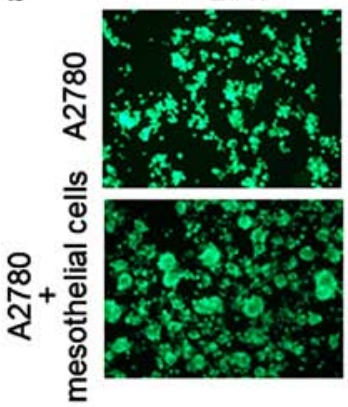

c

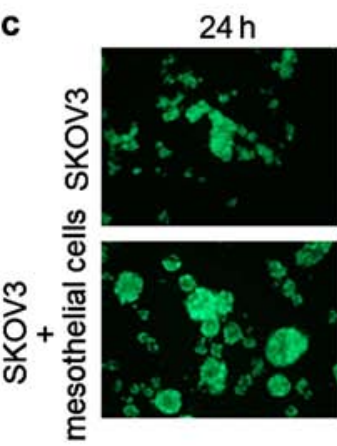

d

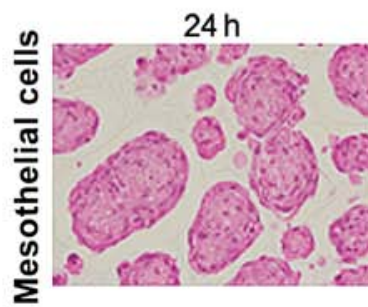

$48 \mathrm{~h}$

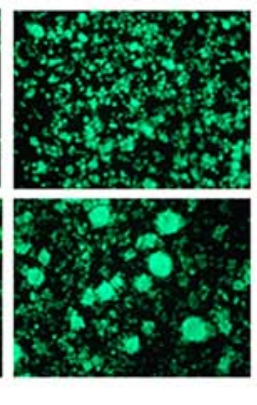

$48 \mathrm{~h}$
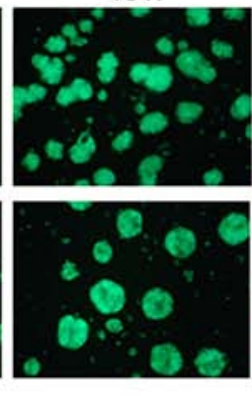

$72 \mathrm{~h}$

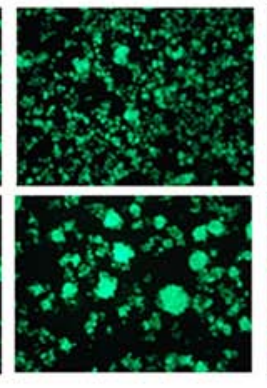

$72 \mathrm{~h}$
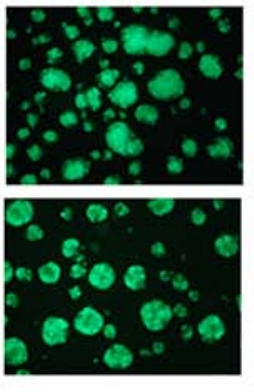

$120 \mathrm{~h}$
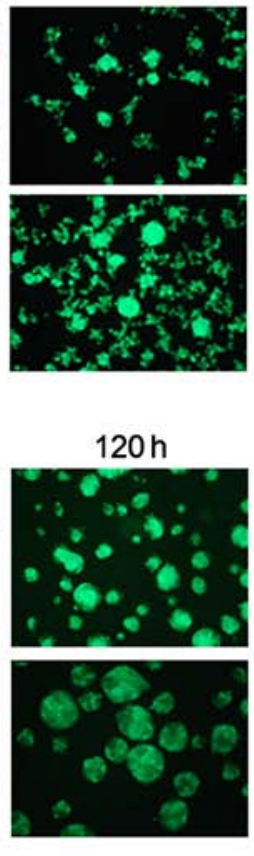

$72 \mathrm{~h}$
$48 h$

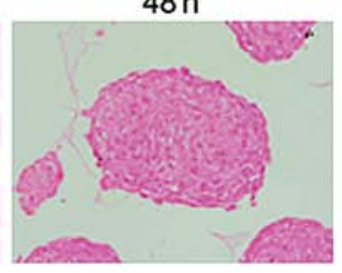

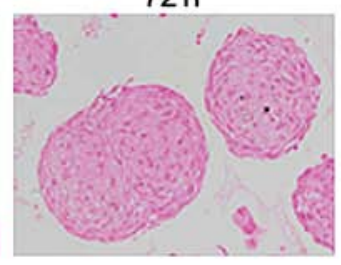

Figure 2. Spheroid formation by ovarian cancer cell lines in the presence or absence of rat mesothelial cells. (A) Three ovarian cancer cell lines, CAOV3, A2780 and SKOV3, were stably labeled with green fluorescence protein (GFP) and cultured with or without rat primary mesothelial cells for $72 \mathrm{~h}$ in suspension. All three cell lines formed spheroids in the presence of mesothelial cells (lower panels), whereas the SKOV3 cells formed the spheroids even without mesothelial cells (upper panels). Magnification, x100. (B) Time course of spheroid formation in (a) CAOV3, (b) A2780, and (c) SKOV3 cells. Magnification, x100; (d) H\&E staining of rat mesothelial cells alone. Magnification, $\mathrm{x} 400$. 
A

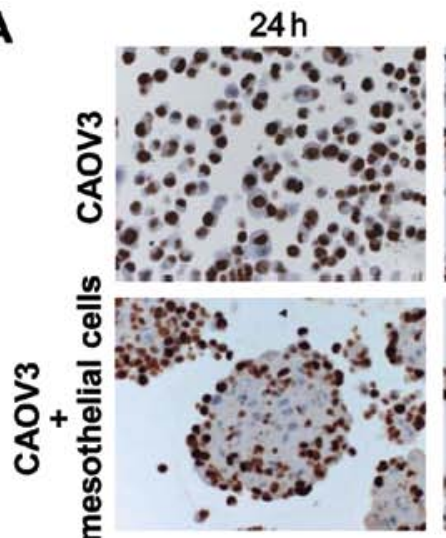

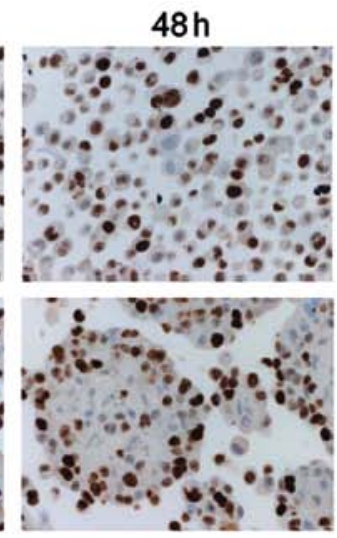

$48 \mathrm{~h}$

B
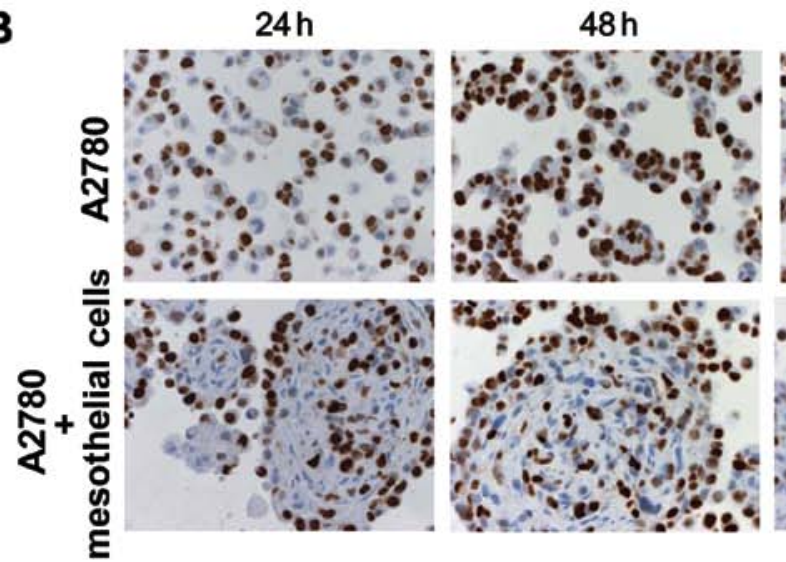
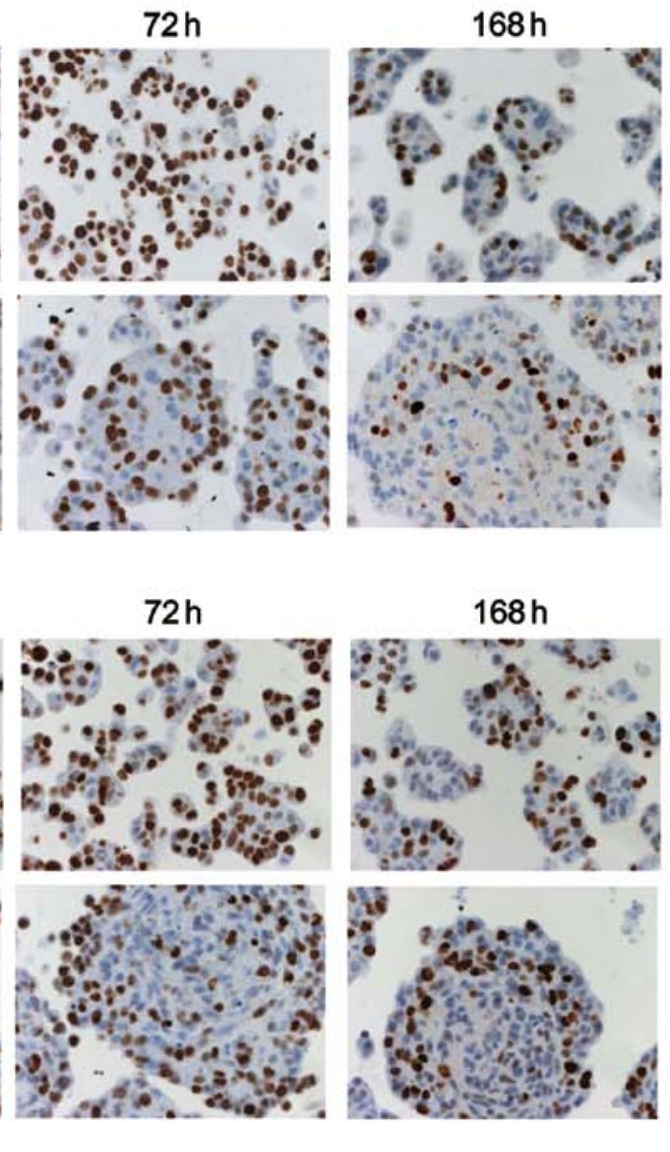

C
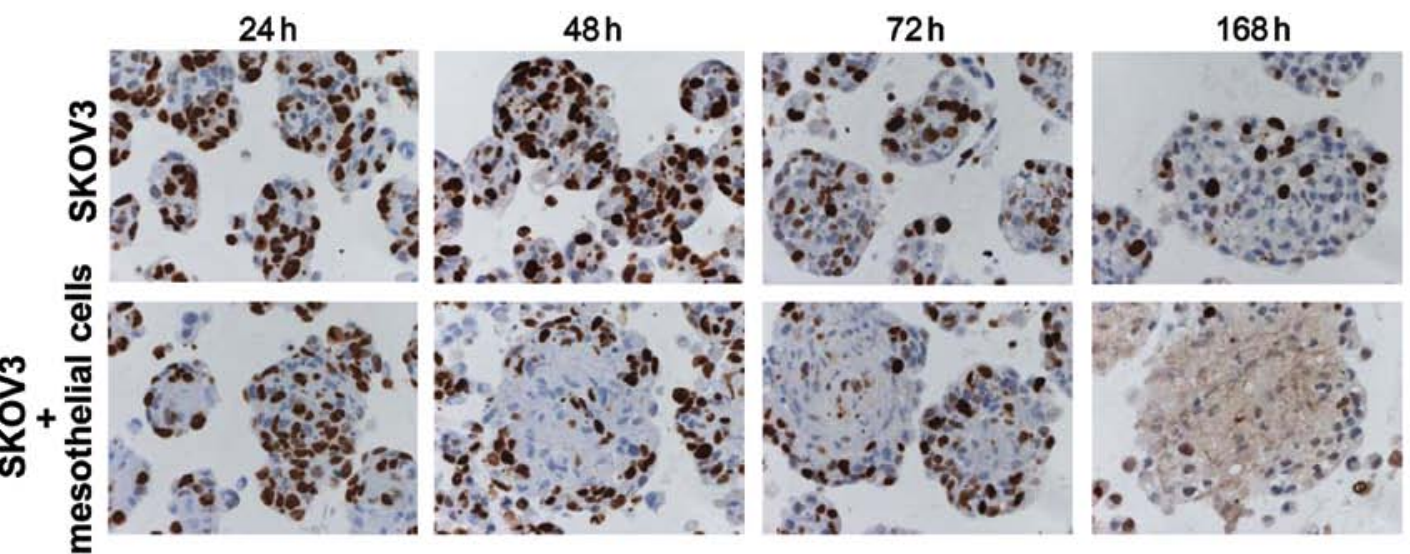

Figure 3. Ki-67 immunostaining in spheroids. Ki-67-positive cells were located in the peripheral region of the spheroids of each cell type, namely (A) CAOV3, (B) A2780 and (C) SKOV3, especially after $72 \mathrm{~h}$ in culture. SKOV3 cells alone could form spheroids and Ki-67-positive cells were randomly distributed in spheroids (upper panels of C). Magnification, x200.

Similar CSC marker expression was observed in A2780 cells (Fig. 4C).

Effect of mesothelial cells on cancer stem cell marker mRNA expression. Subsequently, we examined the mRNA levels of the stem cell markers in CAOV3 cells using human-specific primers for CD44s, Dclk-1 and Bmi-1, which do not detect mRNA derived from rat mesothelial cells. We observed that CD44s and Dclk-1 transcripts significantly increased in co-culture with mesothelial cells at $48 \mathrm{~h}$ compared to CAOV3 cells alone $(\mathrm{P}<0.01$ and $\mathrm{P}<0.05$, respectively; Fig. $5 \mathrm{~A}$ and $\mathrm{B})$. Conversely, Bmi-1 mRNA did not increase at $48 \mathrm{~h}$, but significantly increased at $96 \mathrm{~h}$ in co-culture with mesothelial cells $(\mathrm{P}<0.05$, Fig. 5C).

Mesothelial cells promote in vivo ovarian cancer growth in xenografts. Subcutaneous injection of the mixture of CAOV3 and mesothelial cells resulted in tumors on day 25 in 7 of 7 mice (100\%). Subcutaneous tumors derived from CAOV3 cells alone were detected in 4 of 7 mice $(57 \%)$ on day 36 . Injection of mesothelial cells did not produce tumors in the three injected mice. The tumor volume was significantly larger in the CAOV3 and mesothelial cell group than the CAOV3 alone group $(\mathrm{P}<0.0001$, Fig. 6). The tumors had a solid 
Aa

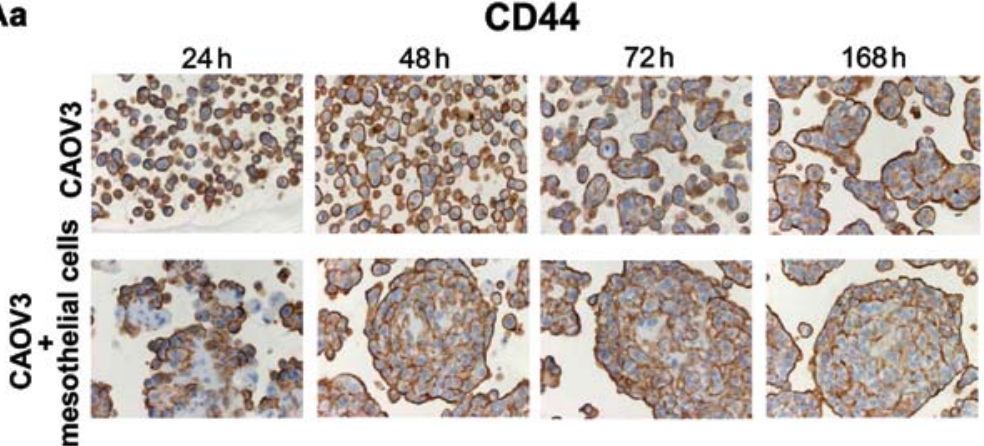

b

ALDH1/2

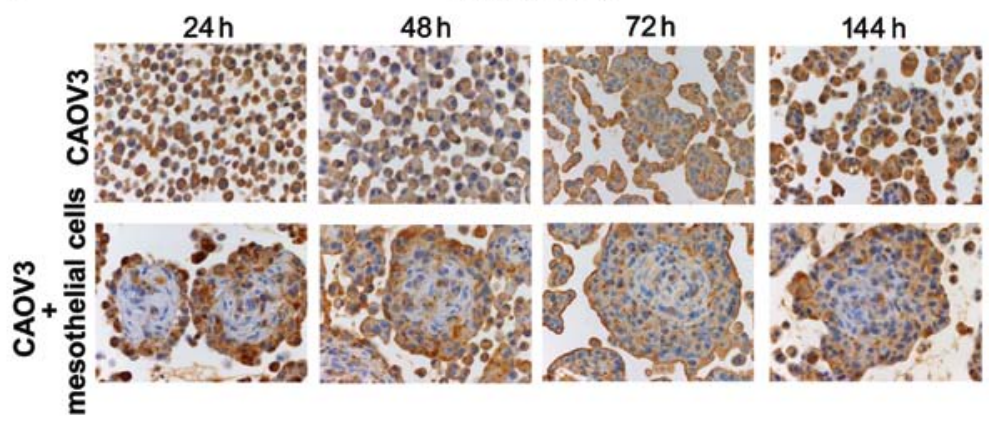

C

\section{CD133}
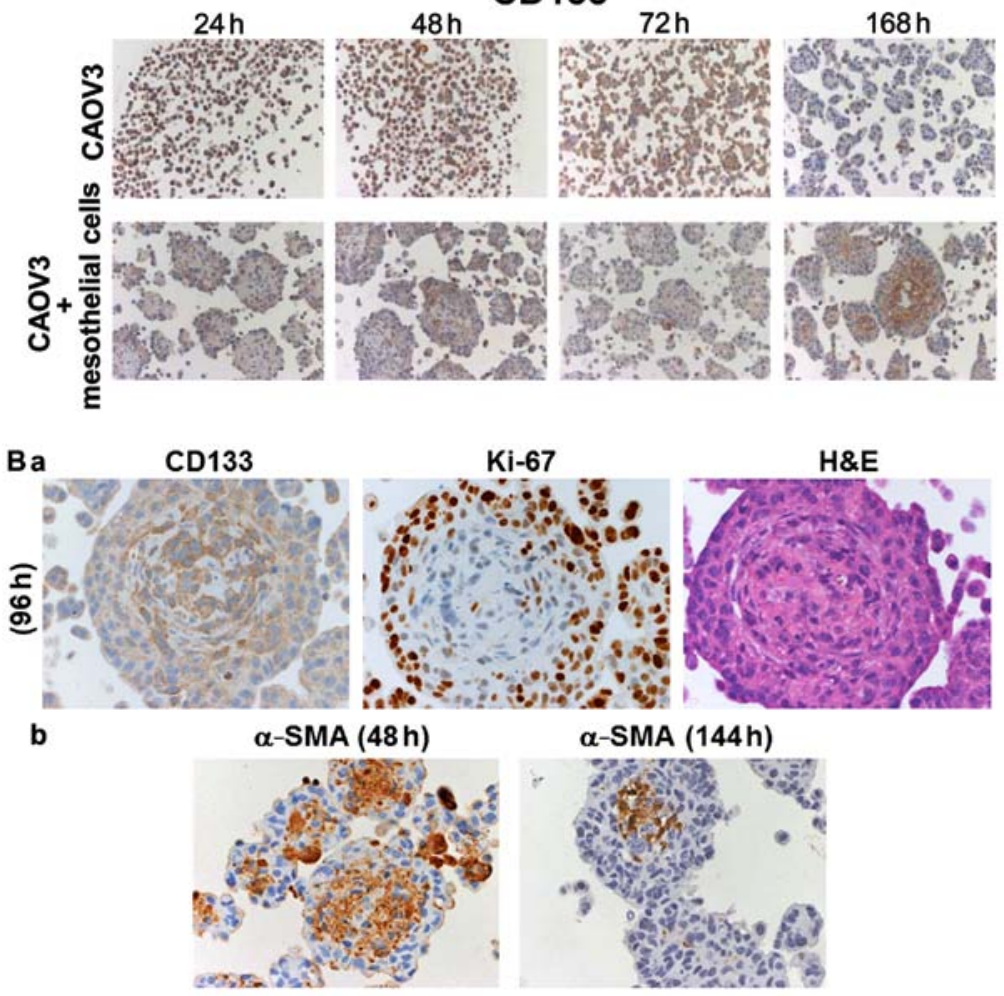

$\mathrm{Ki}-67$

H\&E

c

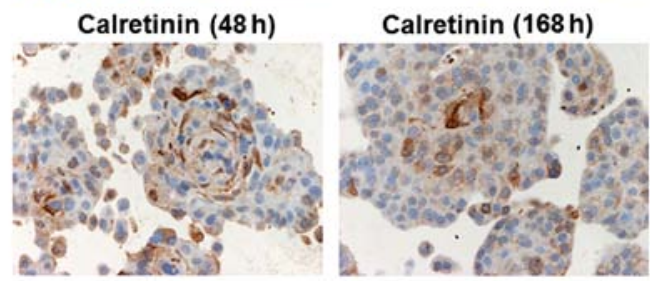

Figure 4. Immunostaining of stem cell markers. (A) Stem cell markers in cell blocks of CAOV3 spheroids stained for (a) CD44, (b) ALDH1/2 and (c) CD133. Expression of CD44 and ALDH1/2 was observed at the periphery of spheroids after $24 \mathrm{~h}$ in co-culture with mesothelial cells, and then extended to the whole spheroid body at subsequent time-points. The expression of CD133 was weak but increased at the inner portion of the spheroids at later time-points in co-culture with mesothelial cells. Magnification, x200 for CD44 and ALDH1/2; magnification, x100 for CD133. (B) Comparison of CD133 and Ki-67 staining and the distribution of mesothelial cells in CAOV3 spheroids. (a) Intense expression of CD133 was observed in the cell membrane at the inner portion of spheroids at $96 \mathrm{~h}$, whereas Ki-67 staining was found at the periphery of spheroids. (b) Mesothelial cells with the $\alpha$-SMA antibody were located in the central area at $48 \mathrm{~h}$ and later shrunk (144 h). (c) Calretinin staining of mesothelial cells demonstrated concordant results at 48 and $168 \mathrm{~h}$. Magnification, x400. 
$\mathbf{C a}$
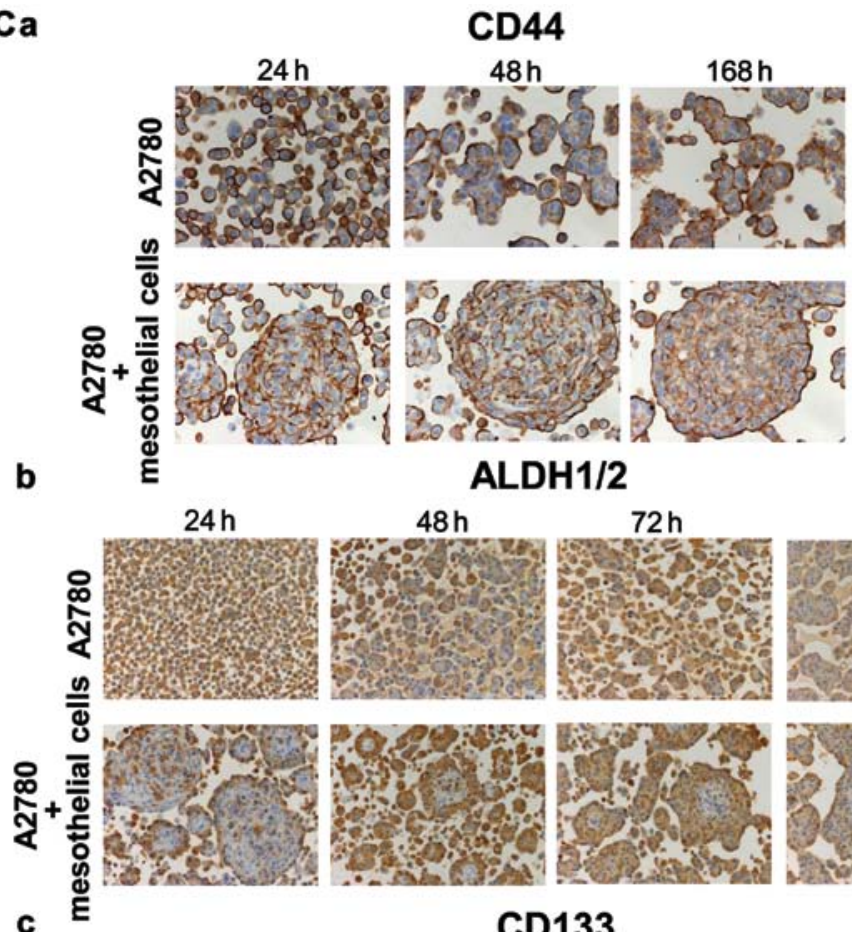

$72 \mathrm{~h}$ $144 \mathrm{~h}$

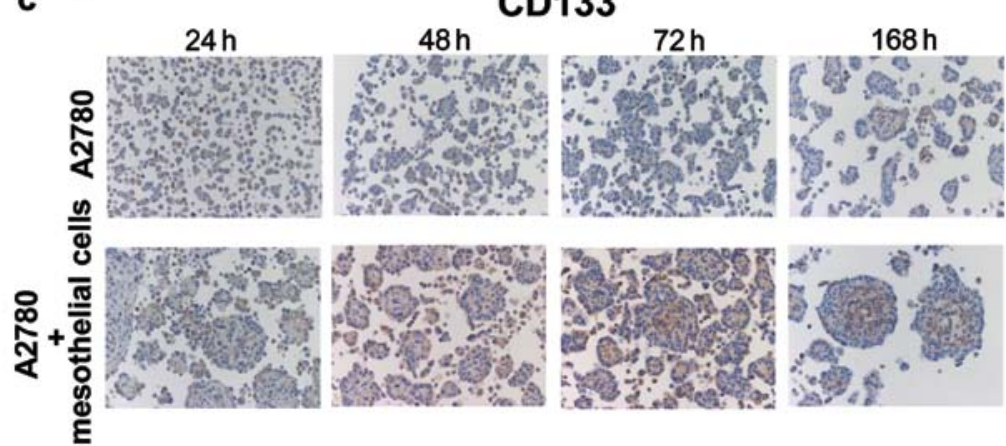

Figure 4. Continued. (C) Stem cell markers in cell blocks of A2780 spheroids stained for (a) CD44, (b) ALDH1/2 and (c) CD133. Expression of CD44 and ALDH1/2 was observed at the periphery of spheroids at $24 \mathrm{~h}$ in co-culture with mesothelial cells and then extended to the whole spheroid body at subsequent time-points. The expression of CD133 was weak but increased at the inner portion of spheroids at subsequent time-points in co-culture with mesothelial cells. Magnification, x200 for CD44 and ALDH1/2; magnification, x100 for CD133.

histological pattern and no significant morphological difference was observed between the two CAOV3 groups (data not shown).

Spheroid formation by ovarian cancer cells in the presence of human mesothelial cells. Finally, we used human Met-5A mesothelial cells instead of rat mesothelial cells. Three ovarian cancer cell lines, CAOV3, A2780 and SKOV3, were stably labeled with GFP and cultured with human Met-5A mesothelial cells for $48 \mathrm{~h}$ in suspension. All three ovarian cancer cell lines formed spheroids in the presence of mesothelial cells (Fig. 7).

\section{Discussion}

During daily workups for cytopathological diagnosis, the first author of the present study, noticed morphological features in H\&E staining indicating that the mesothelial cells were often mixed in the cancerous cluster balls called 'spheroids' in the ascites of ovarian cancer patients. The presence of mesothelial cells was ascertained in clinical spheroids based on calretinin or $\alpha$-SMA staining. The present study was performed to address her long-time question regarding the role of mesothelial cells in ovarian cancer spheroids.

In GFP-tagged 3D-culture studies, we observed that co-culturing ovarian cancer cells and rat mesothelial cells facilitated spheroid formation. In CAOV3 and A2780 cells, significantly larger spheroids were produced by the mixture of mesothelial cells. In contrast, SKOV3 cells formed spheroids without mesothelial cells, as previously reported (21). The SKOV3 spheroids were reported to express a high level of cancer stem markers, such as CD133 and CD117. Nevertheless, it is of note that the initial spheroid formation at 24-48 h appeared to be facilitated by mesothelial cells (Fig. 2B-c). Recent molecular-based analysis has uncovered that the high grade serous ovarian carcinoma is of fimbriae tubae origin (22). Furthermore, the genetic expression profile of SKOV3 cells was different from that of other serous carcinoma types (23-25). This may explain, at least in part, the enhanced spheroid formation ability of SKOV3 cells.

Long-term 3D-culture is one of the characteristics of the present study. Repeated experiments indicated that the spheroids generated in co-cultures appeared to be healthier 

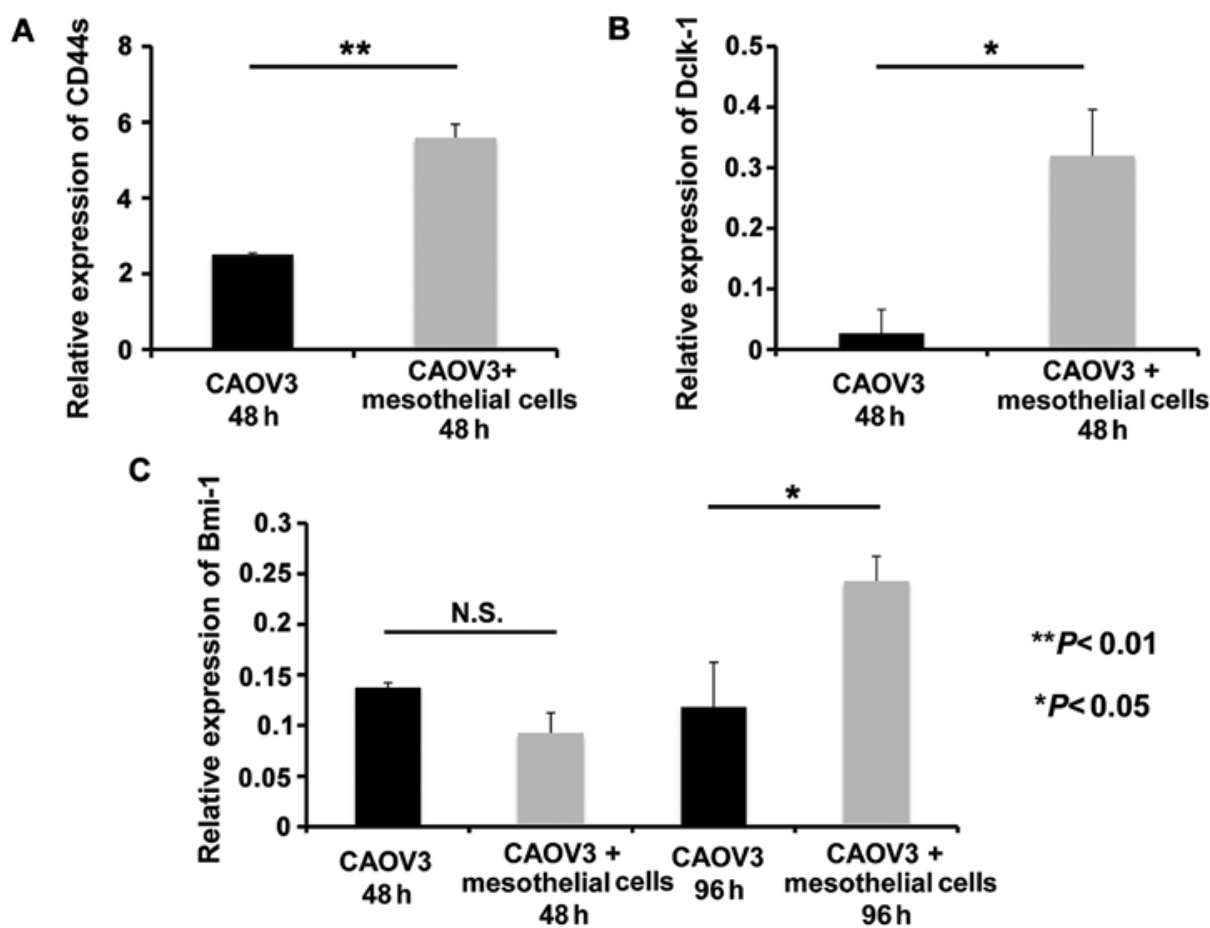

Figure 5. Quantitative analysis of the stem cell markers in spheroids. CAOV3 cells were cultured with or without mesothelial cells in suspension. (A) The expression of stem cell markers CD44s, (B) Dclk-1 and (C) Bmi-1 was assessed in spheroids by qRT-PCR. The expression of CD44s and Dclk-1 was higher in co-culture with mesothelial cells than cancer cells alone at $48 \mathrm{~h}(\mathrm{P}<0.01, \mathrm{P}<0.05$, respectively). In contrast, the expression of Bmi-1 was similar in co-culture with mesothelial cells as in cancer cells alone at $48 \mathrm{~h}$, but was higher at $96 \mathrm{~h}(\mathrm{P}<0.05) .{ }^{*} \mathrm{P}<0.05,{ }^{* *} \mathrm{P}<0.01 ; \mathrm{NS}$, not significant.

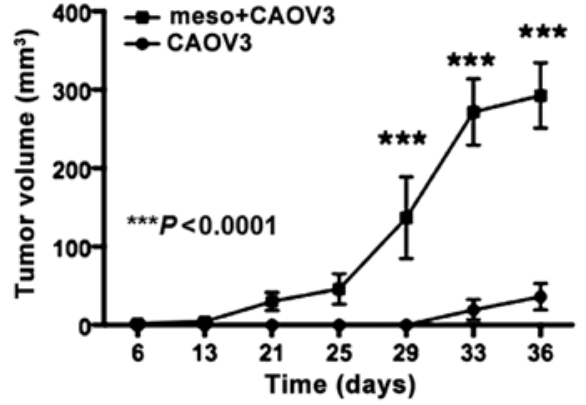

Figure 6. Tumorigenicity of spheroids in vivo. CAOV3 cells were cultured with or without mesothelial cells for $24 \mathrm{~h}$ in suspension, and then injected into mice subcutaneously. The tumor volume was significantly larger in the CAOV3 + mesothelial cell group than the CAOV3 alone group. ${ }^{* * *} \mathrm{P}<0.0001$. meso + CAOV3, mesothelial cells + CAOV3 .

after $120 \mathrm{~h}$ of culture compared to those produced in monocultures (Fig. 2B). Based on the findings, we hypothesized that such spheroids may be endowed with cancer stemness.

Experiments on the proliferative activity assessed by Ki-67 staining revealed a specific proliferation pattern of the co-cultured spheroids. Cell block studies revealed that $\mathrm{Ki}$-67-positive cells were located at the peripheral region of spheroids in each cell type, especially after $72 \mathrm{~h}$ in culture. This contrasted with the random distribution of Ki-67-stained cells in the spheroids consisting of SKOV3 cells alone (Fig. 3C). When we examined the localization of mesothelial cells, we observed that they stayed in the central portion of spheroids. These findings indicated that tumor cells at the periphery should have high proliferative activity in spheroids produced from co-culture.
Spheroid or sphere formation is one of the hallmarks of CSCs. Bone morphogenetic protein (BMP) 2 promoted the phosphorylation of SMAD5 and induced epithelial-mesenchymal transition (EMT) (26). Taking into consideration that the CAOV3 and A2780 cells exhibited prominent spheroid formation with the aid of mesothelial cells, we examined the expression of the cell surface markers of CSCs. Since the majority of CAOV3 and A2780 cells initially expressed CD44 and ALDH1/2, time-course studies indicated that tumor cells expanded from the periphery to the central area. For example, CD44-positive cells were partially located at the periphery at $24 \mathrm{~h}$, continued to grow, and occupied almost the entire spheroid at $168 \mathrm{~h}$. This was consistent with the observation that mesothelial cells residing in the central area gradually disappeared.

CD133 (also known as prominin-1) is a putative cell surface marker for ovarian CSCs and other types of solid tumors $(27,28)$. One of the major findings of the present study was that CD133-positive cancer cells emerged in the late phase (72-168 h) in the inner portion of the spheroids of CAOV3 and A2780 cells. Since this antibody reacted with the human CD133 membranous protein $(29,30)$, and based on the morphological assessment of epithelial-like cells in the high-power image, the CD133-expressing cells were tumor cells, probably arresting at the G0-G1 phase of the cell cycle. The expression of CD133 was consistently reported to be associated with the CSC population at the G0/G1 phase in osteosarcoma and gastric cancer $(31,32)$. Bmi-1 and Lrig-1 are known quiescent stem markers, which contrast active CSC markers, such as Lgr5 and Dclk-1 (33-36). Notably, RNA expression of CSC markers CD44s and Dclk-1 was 

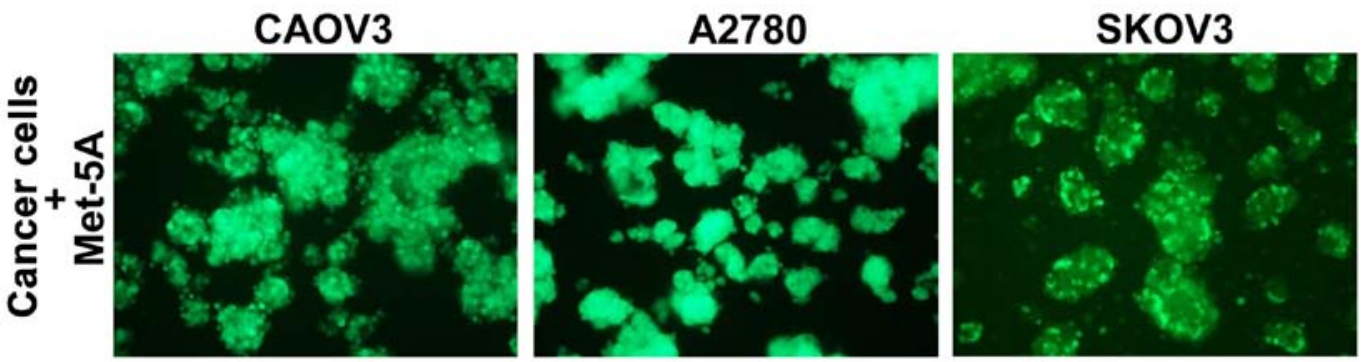

Figure 7. Spheroid formation by ovarian cancer cells in the presence of human mesothelial cells. Three ovarian cancer cell lines, CAOV3, A2780 and SKOV3, were stably labeled with GFP and cultured with human Met-5A mesothelial cells for $48 \mathrm{~h}$ in suspension. All three ovarian cancer cell lines formed spheroids in the presence of mesothelial cells. Magnification, x100. GFP, green fluorescent protein.

increased at $48 \mathrm{~h}$ in co-culture, and Bmi-1 mRNA increased relatively late (i.e. $96 \mathrm{~h}$ ), when the CAOV3 tumor cells were positive for CD133 expression. In animal experiments, we observed that co-cultured cells exhibited enhanced tumorigenicity (7/7 vs. 4/7) and a significant increase in tumor volume compared to CAOV3 monoculture. High tumorigenicity is the best hallmark of CSCs, indicating that co-cultured cells acquire cancer stem-like properties.

Our findings indicated a scenario for the genesis and survival of spheroids in carcinomatous peritonitis from ovarian cancer. With the mesothelial cells in ascites as the core, ovarian cancer cells attached to it, possibly via the adhesion mechanism of $\alpha 2 \beta 1, \alpha 5 \beta 1, \alpha 3 \beta 1, \alpha v \beta 1$ or $\alpha 6 \beta 1$ integrins, hyaluronic acid or CD44 (14,37-39). Tumor cells gradually grew and expanded from the periphery to the central area of the spheroid body. After quiescent CSCs were generated in the inner region, the spheroid acquired eternal survival, through the continuous self-renewal of CSCs and asymmetric division, producing differentiated daughter cells. At the later phase, the initially required mesothelial cells were no longer necessary. Furthermore, one of the reasons for disease recurrence after chemotherapy was assumed to be due to the presence of CSCs in spheroids.

In the present study, we used rat mesothelial cells, which may raise a concern about xenogenic (interspecies) reactions in the process of spheroid formation, and this is a possibility that cannot be denied. Prior to the study, we confirmed that human Met-5A mesothelial cells can make spheroids in cooperation with ovarian cancer cells (Fig. 7). However, we considered that immortalized Met-5A cells transfected with pRSV-T plasmid containing the SV40 early region and Rous sarcoma virus long terminal repeat may not be suitable for analyzing the physiological interaction between tumor cells and non-tumor mesothelial cells. To emphasize non-tumor features, we eventually decided to prepare rat mesothelial cells from the omentum and mesenterium, when they would be necessary. We observed that such non-tumor cells were going away from spheroids after a long time in culture, whereas tumor cells gradually expanded and survived long-term with the acquisition of cancer stem-like properties. With immortal Met-5A cells, this dynamic physiological reaction may have proceeded differently.

In addition, it is important to investigate whether mesothelial cells are located at the central portion of spheroids in clinical samples. During daily workups for cytopathological diagnosis, it is demanding to examine the parts inside of spheroids, since cell block is required for this purpose. In addition, since spheroids are not common among the various cytological specimens, a confirmation study may be considered as a future research direction.

In conclusion, the present study provided a novel in vitro 3D spheroid model of ovarian cancer cells with rat mesothelial cells. Our results revealed that mesothelial cells enhanced stem cell-related gene expression and facilitated spheroid formation in 3D-culture and tumor formation in a xenograft model, which is considered a hallmark of CSCs. Further studies of carcinomatous peritonitis using clinically collected mesothelial cells are essential in order to fully comprehend the pathogenesis of this disease from one stage to the next.

\section{Acknowledgements}

Not applicable.

\section{Funding}

The present study was supported by a Grant-in-Aid for Scientific Research (grant no. 17K08775) from the Japan Society for the Promotion of Science (to SM). The funders had no role in the study design, data collection and analysis, decision to publish, or preparation of the manuscript.

\section{Availability of data and materials}

The datasets used during the present study are available from the corresponding author upon reasonable request.

\section{Authors' contributions}

AS, SM and HY designed the study. AS, SM, YY, KM, YQ, JW, HH and XW performed the experiments. AS, SM, YY and HY analyzed the data and compiled the figures. AS, SM and HY wrote the manuscript. YH, NK, SN and NM reviewed and edited the manuscript and also participated in the conception of the study. All authors read and approved the contents of the manuscript and agree to be accountable for all aspects of the research in ensuring that the accuracy or integrity of any part of the work are appropriately investigated and resolved.

\section{Ethics approval and consent to participate}

All experiments using mice were approved by the Institutional Animal Care and Use Committee of Osaka University 
Graduate School of Medicine and the Committee for the Ethics of Animal Experiments of Osaka University (approval nos. 28-03-001; Osaka, Japan).

\section{Consent for publication}

Not applicable.

\section{Competing interests}

The authors state that they have no competing interests.

\section{References}

1. Ozols RF: Systemic therapy for ovarian cancer: Current status and new treatments. Semin Oncol 33 (2 Suppl 6): S3-S11, 2006.

2. Liao J, Qian F, Tchabo N, Mhawech-Fauceglia P, Beck A, Qian Z, Wang X, Huss WJ, Lele SB, Morrison CD, et al: Ovarian cancer spheroid cells with stem cell-like properties contribute to tumor generation, metastasis and chemotherapy resistance through hypoxia-resistant metabolism. PLoS One 9: e84941, 2014.

3. Foster R, Buckanovich RJ and Rueda BR: Ovarian cancer stem cells: Working towards the root of stemness. Cancer Lett 338: 147-157, 2013.

4. Shield K, Ackland ML, Ahmed N and Rice GE: Multicellular spheroids in ovarian cancer metastases: Biology and pathology Gynecol Oncol 113: 143-148, 2009.

5. Burleson KM, Boente MP, Pambuccian SE and Skubitz AP: Disaggregation and invasion of ovarian carcinoma ascites spheroids. J Transl Med 4: 6, 2006.

6. Shen-Gunther J and Mannel RS: Ascites as a predictor of ovarian malignancy. Gynecol Oncol 87: 77-83, 2002.

7. Ayantunde AA and Parsons SL: Predictors of poor prognosis in patients with malignant ascites: A prospective study. Clin Med Diagnostics 2: 1-6, 2012.

8. Mackey JR and Venner PM: Malignant ascites: Demographics, therapeutic efficacy and predictors of survival. Can J Oncol 6: 474-480, 1996.

9. Mills GB, May C, Hill M, Campbell S, Shaw P and Marks A: Ascitic fluid from human ovarian cancer patients contains growth factors necessary for intraperitoneal growth of human ovarian adenocarcinoma cells. J Clin Invest 86: 851-855, 1990.

10. Mutsaers SE: Mesothelial cells: Their structure, function and role in serosal repair. Respirology 7: 171-191, 2002.

11. Lengyel E: Ovarian cancer development and metastasis. Am J Pathol 177: 1053-1064, 2010.

12. Burleson KM, Casey RC, Skubitz KM, Pambuccian SE, Oegema TR Jr and Skubitz AP: Ovarian carcinoma ascites spheroids adhere to extracellular matrix components and mesothelial cell monolayers. Gynecol Oncol 93: 170-181, 2004.

13. Strobel $\mathrm{T}$ and Cannistra SA: Beta1-integrins partly mediate binding of ovarian cancer cells to peritoneal mesothelium in vitro. Gynecol Oncol 73: 362-367, 1999.

14. Mikuła-Pietrasik J, Sosińska P and Książek K: Resveratrol inhibits ovarian cancer cell adhesion to peritoneal mesothelium in vitro by modulating the production of $\alpha 5 \beta 1$ integrins and hyaluronic acid. Gynecol Oncol 134: 624-630, 2014.

15. Cannistra SA, Kansas GS, Niloff J, DeFranzo B, Kim Y and Ottensmeier C: Binding of ovarian cancer cells to peritoneal mesothelium in vitro is partly mediated by CD44H. Cancer Res 53: 3830-3838, 1993.

16. Visvader JE and Lindeman GJ: Cancer stem cells in solid tumours: Accumulating evidence and unresolved questions. Nat Rev Cancer 8: 755-768, 2008.

17. Skubitz AP, Taras EP, Boylan KL, Waldron NN, Oh S, Panoskaltsis-Mortari A and Vallera DA: Targeting CD133 in an in vivo ovarian cancer model reduces ovarian cancer progression. Gynecol Oncol 130: 579-587, 2013.

18. Akedo H, Shinkai K, Mukai M, Mori Y, Tateishi R, Tanaka K, Yamamoto R and Morishita T: Interaction of rat ascites hepatoma cells with cultured mesothelial cell layers: A model for tumor invasion. Cancer Res 46: 2416-2422, 1986.

19. Stylianou E, Jenner LA, Davies M, Coles GA and Williams JD: Isolation, culture and characterization of human peritoneal mesothelial cells. Kidney Int 37: 1563-1570, 1990.
20. Doglioni C, Dei Tos AP, Laurino L, Iuzzolino P, Chiarelli C, Celio MR and Viale G: Calretinin: A novel immunocytochemical marker for mesothelioma. Am J Surg Pathol 20: 1037-1046, 1996.

21. Ma L, Lai D, Liu T, Cheng W and Guo L: Cancer stem-like cells can be isolated with drug selection in human ovarian cancer cell line SKOV3. Acta Biochim Biophys Sin 42: 593-602, 2010.

22. Kindelberger DW, Lee Y, Miron A, Hirsch MS, Feltmate C, Medeiros F, Callahan MJ, Garner EO, Gordon RW, Birch C, et al: Intraepithelial carcinoma of the fimbria and pelvic serous carcinoma: Evidence for a causal relationship. Am J Surg Pathol 31: 161-169, 2007.

23. Domcke S, Sinha R, Levine DA, Sander C and Schultz N: Evaluating cell lines as tumour models by comparison of genomic profiles. Nat Commun 4: 2126, 2013.

24. Elias KM, Emori MM, Papp E, MacDuffie E, Konecny GE, Velculescu VE and Drapkin R: Beyond genomics: Critical evaluation of cell line utility for ovarian cancer research. Gynecol Oncol 139: 97-103, 2015.

25. Anglesio MS, Wiegand KC, Melnyk N, Chow C, Salamanca C, Prentice LM, Senz J, Yang W, Spillman MA, Cochrane DR, et al: Type-specific cell line models for type-specific ovarian cancer research. PLoS One 8: e72162, 2013.

26. McLean K, Gong Y, Choi Y, Deng N, Yang K, Bai S, Cabrera L, Keller E, McCauley L, Cho KR and Buckanovich RJ: Human ovarian carcinoma-associated mesenchymal stem cells regulate cancer stem cells and tumorigenesis via altered BMP production. J Clin Invest 121: 3206-3219, 2011.

27. Grosse-Gehling P, Fargeas CA, Dittfeld C, Garbe Y, Alison MR, Corbeil D and Kunz-Schughart LA: CD133 as a biomarker for putative cancer stem cells in solid tumours: Limitations, problems and challenges. J Pathol 229: 355-378, 2013.

28. Ajani JA, Song S, Hochster HS and Steinberg IB: Cancer stem cells: The promise and the potential. Semin Oncol 42 (Suppl 1): S3-S17, 2015.

29. Motegi H, Kamoshima Y, Terasaka S, Kobayashi H and Houkin K: Type 1 collagen as a potential niche component for CD133-positive glioblastoma cells. Neuropathology 34: 378-385, 2014.

30. Okudela K, Woo T, Mitsui H, Tajiri M, Masuda M and Ohashi K: Expression of the potential cancer stem cell markers, CD133, CD44, ALDH1, and $\beta$-catenin, in primary lung adenocarcinoma - their prognostic significance. Pathol Int 62: 792-801, 2012.

31. Yano S, Tazawa H, Hashimoto Y, Shirakawa Y, Kuroda S, Nishizaki M, Kishimoto H, Uno F, Nagasaka T, Urata Y, et al: A genetically engineered oncolytic adenovirus decoys and lethally traps quiescent cancer stem-like cells in S/G2/M phases. Clin Cancer Res 19: 6495-6505, 2013.

32. Li J, Zhong XY, Li ZY, Cai JF, Zou L, Li JM, Yang T and Liu W: CD133 expression in osteosarcoma and derivation of CD133+ cells. Mol Med Rep 7: 577-584, 2013.

33. Sangiorgi E and Capecchi MR: Bmil is expressed in vivo in intestinal stem cells. Nat Genet 40: 915-920, 2008.

34. Powell AE, Wang Y, Li Y, Poulin EJ, Means AL, Washington MK, Higginbotham JN, Juchheim A, Prasad N, Levy SE, et al: The pan-ErbB negative regulator Lrig1 is an intestinal stem cell marker that functions as a tumor suppressor. Cell 149: 146-158, 2012.

35. Barker N, van Es JH, Kuipers J, Kujala P, van den Born M, Cozijnsen M, Haegebarth A, Korving J, Begthel H, Peters PJ and Clevers $\mathrm{H}$ : Identification of stem cells in small intestine and colon by marker gene Lgr5. Nature 449: 1003-1007, 2007.

36. Nakanishi Y, Seno H, Fukuoka A, Ueo T, Yamaga Y, Maruno T, Nakanishi N, Kanda K, Komekado H, Kawada M, et al: Dclk1 distinguishes between tumor and normal stem cells in the intestine. Nat Genet 45: 98-103, 2013.

37. Ahmed N, Riley C, Rice G and Quinn M: Role of integrin receptors for fibronectin, collagen and laminin in the regulation of ovarian carcinoma functions in response to a matrix microenvironment. Clin Exp Metastasis 22: 391-402, 2005.

38. Shield K, Riley C, Quinn MA, Rice GE, Ackland ML and Ahmed N: Alpha2beta1 integrin affects metastatic potential of ovarian carcinoma spheroids by supporting disaggregation and proteolysis. J Carcinog 6: 11, 2007.

39. Sawada K, Mitra AK, Radjabi AR, Bhaskar V, Kistner EO, Tretiakova M, Jagadeeswaran S, Montag A, Becker A, Kenny HA, et al: Loss of E-cadherin promotes ovarian cancer metastasis via alpha 5-integrin, which is a therapeutic target. Cancer Res 68: 2329-2339, 2008. 